\title{
A TRANSIÇÃO EPIDEMIOLÓGICA NO ESTADO DE MATO GROSSO DO SUL, BRASIL: O CASO DOS ÓBITOS POR ALGUMAS DOENÇAS INFECCIOSAS E PARASITÁRIAS E NEOPLASIAS NOS ANOS DE 2007 A 2017
}

\section{THE EPIDEMIOLOGICAL TRANSITION IN THE MATO GROSSO DO SUL STATE, BRAZIL: DEATH EVENTS BY SOME INFECTIOUS AND PARASITIC DESEASES AND NEOPLASIA BETWEEN THE YEARS OF 2007 AND 2017}

\author{
Geovandir André Lordano \\ Programa de Pós-Graduação em Geografia - PPGGEO/CPAQ \\ Universidade Federal de Mato Grosso do Sul - UFMS, Brasil \\ geovandirlordano@gmail.com \\ Daiane Santana Santos \\ Programa de Pós-Graduação em Geografia - PPGGEO/CPAQ \\ Universidade Federal de Mato Grosso do Sul - UFMS, Brasil \\ daianesantanageo@gmail.com \\ Eva Teixeira dos Santos \\ Programa de Pós-Graduação em Geografia - PPGGEO/CPAQ \\ Universidade Federal de Mato Grosso do Sul - UFMS, Brasil \\ evasantos.ufms@gmail.com
}

\begin{abstract}
RESUMO
Esta pesquisa tem como objetivo analisar no contexto do estado de Mato Grosso do Sul Brasil, a ocorrência dos óbitos por doenças pertencentes aos grupos: CID-10: A00 - B99 Algumas doenças infecciosas e parasitárias; e C00-D48 - Neoplasias, nos anos de 2007 a 2017. A metodologia é a de pesquisa descritiva, utilizando-se da técnica de pesquisa documental e eletrônica sobre o tema e sobre o objeto analisado. Os dados secundários são fornecidos pela plataforma TabNet, da Secretária de Estado de Saúde de Mato Grosso do Sul. Após análise dos dados, tem-se que o caso de óbitos por doenças do grupo CID-10: A00 - B99 - Algumas doenças infecciosas e parasitárias - teve um declínio de 9,59\% no coeficiente de mortalidade e redução de $9,90 \%$ em seu peso entre o número total de óbitos no estado. Já o grupo CID-10 C00-D48 - Neoplasias - apresenta um aumento de 23,67\% no coeficiente de mortalidade e $23,13 \%$ em seu peso entre o total de óbitos em Mato Grosso do Sul. Logo, pode-se concluir que o estado sul-mato-grossense se encontra em um processo de transição epidemiológica, com considerável variação em seu perfil de mortalidade, pressionada pela significativa alta da ocorrência das neoplasias no período, evidenciando a necessidade de políticas em saúde destinadas ao novo perfil de mortalidade.
\end{abstract}

Palavras-Chave: TabNet. Perfil de Mortalidade. Transição Demográfica. Condições Sanitárias. Agrotóxicos.

\begin{abstract}
This research aims to analyse within the Mato Grosso do Sul State - Brazil context the occurrence of death by diseases from groups: CID-10: A00 - B99; some infectious and parasitic diseases; and C00-D48 - Neoplasia, between de years of 2007 and 2017. As methodology was used descriptive research, using the technique of documental and electronic research on the subject and the analysed object. The secondary data was provided by TabNet interface of the Mato Grosso do Sul State Health Bureau. Having examined the data, it seems that death events by diseases from group CID-10: A00 - B99some infectious and parasitic diseases - have had a decrease of $9,59 \%$ of mortality coefficient and $9,90 \%$ in its weight among the total number of deaths in the state. On the other hand, the group CID-10 C00-D48 - Neoplasia - shows an increase of $23,67 \%$ of
\end{abstract}

Recebido em: 28/02/2019

Aceito para publicação em: 22/02/2020 
mortality coefficient and $23,13 \%$ in its weight among the total number of deaths in Mato Grosso do Sul State. Therefore, it can be concluded that the Mato Grosso do Sul State is currently in a process of epidemiological transition, with significant variance in its mortality profile, pushed by the substantial increase of neoplasia in the period, emphasising the necessity of health policies for its new mortality profile.

Keywords: TabNet. Mortality Profile. Demographic Transition. Sanitary Conditions. Pesticides.

\section{INTRODUÇÃO}

A análise dos padrões de mortalidade de determinada região pode ser considerada por várias áreas do conhecimento, como: medicina, epidemiologia ou pela própria geografia. Nesse contexto, a geografia da saúde é a área da geografia que se dedica à abordagem desses padrões e suas alterações, possibilitando não apenas uma análise em uma perspectiva problematizada, mas também oferecendo subsídios à implantação de políticas de prevenção e acompanhamento em saúde.

A alteração desses padrões de mortalidade é conhecida como transição epidemiológica. Autores como Lebrão (2007) e Schramm et. al. (2004), ambos se referindo à obra de Frenk et al. (1991), apresentam que o conceito de transição epidemiológica faria parte de um conceito maior, isto é, o de transição da saúde, o qual abarcaria aspectos básicos da saúde da população configurando-se em duas vertentes: a primeira em referência à transição das condições de saúde (os processos de saúde-doença que definem o próprio perfil epidemiológico de determinada população, expresso por meio de morte, doença e incapacidades); a segunda, ligada à capacidade de resposta social organizada a essas condições, as quais se materializam por meio do sistema de atenção à saúde. Porém, dada a complexidade que tal temática demandaria, esta pesquisa reserva-se a debater apenas transição epidemiológica.

Sendo assim, a transição epidemiológica, em geral, pode ser conceituada como a modificação no perfil de morbi/mortalidade da população de determinada região advinda das alterações demográficas, econômicas, sociais e de estratégicas de saúde pública que possibilitaram a queda na ocorrência de grupos de doenças características do subdesenvolvimento e da precariedade nele encontrada (PEREIRA; ALVES-SOUZA; VALE, 2015; PRATA, 1992). Isso ocorre da mesma forma em que esse novo padrão proporciona o aumento da incidência de doenças crônico-degenerativas, habitualmente relacionadas às regiões que apresentam melhores condições sanitárias e maior expectativa de vida, comuns aos países que apresentam níveis de desenvolvimento mais consolidados.

Sendo assim, frente à emergência da discussão sobre a modificação dos padrões de mortalidade em parte considerável das regiões subdesenvolvidas e/ou em desenvolvimento, torna-se oportuna a abordagem do tema no contexto do estado de Mato Grosso do Sul, Brasil. Logo, esta pesquisa parte da hipótese de que o estado esteja em um processo de transição de seu padrão de mortalidade, tendo diminuído os casos de óbitos por doenças infecciosas ou parasitárias (típicas de regiões mais pobres e com condições sanitárias mais precárias), passando a apresentar uma incidência maior de óbitos por doenças pertencentes a grupos de doenças mais associadas ao aumento da expectativa de vida, modificação dos hábitos alimentares, sedentarismo e de todas outras alterações advindas do maior desenvolvimento técnico e econômico.

Dessa maneira, este trabalho tem por objetivo geral analisar no contexto do estado de Mato Grosso do Sul - Brasil, a ocorrência dos óbitos por doenças pertencentes aos grupos CID-10: A00 - B99 Algumas doenças infecciosas e parasitárias; e C00-D48 - Neoplasias, nos anos de 2007 a 2017. Como objetivos específicos mostram-se: apresentar, com base na literatura, o conceito de Transição Epidemiológica; realizar uma caracterização do estado de Mato Grosso do Sul; e identificar a alteração no perfil de mortalidade no estado sul-mato-grossense.

Metodologicamente, a pesquisa utiliza o método descritivo, com a técnica de pesquisa bibliográfica para um levantamento da temática abordada, bem como dos aspectos inerentes ao objeto de análise. Da mesma forma, utiliza-se da pesquisa documental e eletrônica para a obtenção dos dados secundários: população e demais caracterizações de Mato Grosso do Sul; e dos óbitos no estado, os DOI:http://dx.doi.org/10.14393/Hygeia153447153 Hygeia 15 (34): 130 -143, Dez./2019 página 130 
quais são disponibilizados pela plataforma TabNet (http://tabnet.saude.ms.gov.br/), diretamente no site da Secretaria de Estado de Saúde de Mato Grosso do Sul, que foram analisados de forma quantitativa. Os coeficientes de mortalidade brutos e padronizados por 100 mil habitantes (número de óbitos no ano / população estimada no ano $X 100.000$ ) foram calculados pelo método direto e, assim como a regressão linear, utilizando a planilha Excel (MICROSOFT OFFICE EXCEL, 2013).

A análise dos anos de 2007 e 2017 foi escolhida por basicamente dois motivos: o primeiro é que, embora para a busca de dados capazes de trazer fundamentos que demonstrem a transição epidemiológica quanto maior o recorte temporal entre as variáveis melhor, os dados disponíveis no sistema Tabnet compreendem o período de 2006 a 2018. Logo, fundamenta-se o segundo motivo: além do recorte temporal estar condicionado à oferta de dados, faz-se necessário que, havendo os dados, exista, ao menos, uma previsão oficial do quantitativo da população do estado pelo IBGE. Assim, com base nas estimativas populacionais do IBGE para os anos de 2007 e 2017, aliando-se à população oficial do ano de 2010 , foram estimadas as populações para os demais anos do período analisado.

Este trabalho justifica-se pela oportunidade da análise do perfil transição epidemiológica, considerando o padrão de mortalidade pelos grupos CID-10 nos anos que abarcam esta pesquisa, fornecendo subsídios a futuras análises mais profundas sobre o assunto, bem como pelas demandas em serviços de saúde que o novo perfil de mortalidade exige do planejamento em saúde.

\section{A CAUSALIDADE DAS DOENÇAS: ASPECTOS INICIAIS}

Tanto a geografia médica clássica como as demais áreas relacionadas à saúde, até meados do século XX, tinham uma visão das doenças e suas causas em uma perspectiva um tanto simplista e uniescalar. A visão da unicausalidade fortalecida pela microbiologia à época pautava seus estudos nos agentes microbiológicos e tinha uma relação de determinação dos ambientes naturais sobre o processo de saúde-doença das populações.

Sobre isto, Lima e Guimarães (2007) pontuam que, desde Hipócrates até meados do século XX, a determinação natural das doenças era um conceito predominante e que não sem razão as doenças infecciosas e parasitárias eram as que mais afligiam a humanidade. De qualquer maneira, reconhecem que há fatores naturais que favoreçam as doenças e recorrem a Sorre (2006) e Megale (1984) para afirmar que:

[...] a dinâmica de complexo patogênico constitui-se a partir dos agentes causais, seus vetores, o meio ambiente e o próprio ser humano que se desenvolve em três planos onde se desenvolve a atividade humana: o plano físico, o plano biológico e o plano social (LIMA; GUIMARÃES, 2007, p. 60).

Logo, afirmam que para cada doença identifica-se uma área que possui determinadas condições ecológico-ambientais localizáveis no tempo e no espaço. Assim, os complexos patogênicos receberiam o nome das doenças às quais correspondem, como o complexo da malária, o complexo da doença de Chagas, entre outros (LIMA; GUIMARÃES, 2007).

Nessa perspectiva, Lima e Guimarães $(2007$, p. 59) recorrem à obra de Sorre (2006) para abordar o conceito de complexo patogênico e assim o conceituam: "[...] o homem é incluído como hospedeiro ou vetor de um agente etiológico que circula entre compartimentos de um sistema ecológico, no qual as necessidades do agente patógeno são satisfeitas e se realimenta".

Contudo, simplificar a incidência de determinadas doenças em determinados espaços como uma questão de determinação ambiental é um tanto inadequado e simplório diante da complexidade à qual a relação saúde-doença manifesta-se frente aos variados contextos pertinentes à relação entre sociedade e natureza.

Dito isso, uma enfermidade não surge ou desaparece como um simples fenômeno natural, sendo o seu surgimento ou a sua desintegração diretamente relacionados com as ações humanas no ambiente. Consequentemente, seria preciso entender o processo de organização e ocupação do espaço geográfico, pelas diferentes sociedades em distintos tempos e lugares, para que se torne possível o entendimento da manifestação das doenças. Isso seria muito importante, pois possibilitaria compreender a gênese das doenças e sua distribuição e, assim, pautar o planejamento de ações de vigilância ambiental em saúde (LIMA; GUIMARÃES, 2007). Nesse contexto, a necessidade de 
compreensão desses aspectos encontra grande respaldo na própria geografia da saúde que surge na emergência de um novo enfoque capaz de romper com o simplismo que a epidemiologia clássica impunha até meados do século XX.

No mesmo período, o uso crescente de fármacos (antibióticos), melhoria nas condições urbanas, campanhas de imunização e de saneamento básico reduziram drasticamente a incidência de doenças infecciosas e parasitárias. Ao passo em que as mortes por essas doenças diminuíam, aumentavam as mortes causadas por doenças crônico-degenerativas - a transição epidemiológica. Contudo, a transição epidemiológica só atingiu a quem pode usufruir dos avanços sociais e tecnológicos advindos do desenvolvimento, da mesma forma que não se pode esperar que o desenvolvimento chegue a todos, existindo não uma determinação ambiental no perfil saúde-doença, mas um processo de determinação social das doenças (LIMA; GUIMARÃES, 2007).

Desta forma, tem-se estabelecido que o contexto econômico e social de uma determinada região - e não apenas o meio ambiente - confere grande pressão na incidência ou não das patologias, evidenciando a importância dos estudos, regionalizados e contextualizados, que a geografia da saúde proporciona.

\section{A TRANSIÇÃO EPIDEMIOLÓGICA E O CONTEXTO NACIONAL}

A modificação do padrão de mortalidade de certa população é, de maneira geral, um evento que deve ser analisado em uma perspectiva abrangente e possibilitando a compreensão dos aspectos que com ela se relacionam, influenciando-a ou sentido seus efeitos.

O perfil da mortalidade das populações é considerado um indicador, de certa forma, relativo, muito por possuir certo grau de sensibilidade e variabilidade. É influenciado, em geral, pelas condições de vida e pelo desenvolvimento de cada população, resultando da interação e da convergência de diversos fatores interdependentes (PEREIRA; ALVES-SOUZA, 2015).

Sendo assim, os modelos de reprodução humana e de produção econômica relacionam-se e determinam a estrutura demográfica e econômica de uma população, como taxa de fertilidade, de mortalidade e de migração, bem como os fatores ambientais e socioculturais devem ser considerados na acepção desse perfil. Logo, é impossível a dissociação entre o nível de mortalidade de sua estrutura e a relação direta com fatores históricos, socioeconômicos, demográficos e ambientais (PEREIRA; ALVES-SOUZA; VALE, 2015; PRATA, 1992).

O declínio do coeficiente geral de mortalidade é uma realidade, porém não é o único fenômeno a ser notado. A diminuição da mortalidade infantil, o aumento da expectativa de vida e a alteração do perfil epidemiológico também são observados nesse fenômeno conhecido como transição epidemiológica (PRATA, 1992).

Na Europa, a modificação do perfil epidemiológico e o declínio da mortalidade deram-se a partir da revolução agrícola e industrial, a partir do século XVIII. A redução das doenças infecciosas advinha da melhoria dos níveis de nutrição e de qualidade de vida proporcionados por tais revoluções somados à crescente melhoria de medidas médicas de prevenção e tratamento e de saneamento básico, no século XX. No entanto, o inverso ocorreu na África, onde, no mesmo período, a expansão colonialista foi responsável pela maior oferta de alimentos na Europa, em detrimento do continente africano, o qual teve modificadas a estrutura produtiva e as culturas tradicionais, da mesma forma em que a alteração da forma de ocupação no continente propiciou a ocorrência constante de epidemias (PRATA, 1992).

Consequentemente, tem-se que a relação saúde-doença não se apresenta como um padrão global, da mesma forma em que a transição no perfil epidemiológico apresenta características peculiares variadas conforme as características de cada continente, país ou região.

Nessa perspectiva, no Brasil, ao longo dos anos, o perfil de morbidade e mortalidade tem sofrido alterações consideráveis e os processos de transição demográfica e epidemiológica resultam na formação de grupos com características peculiares e específicas, como exemplo dos problemas ligados ao envelhecimento da população (PEREIRA; ALVES-SOUZA; VALE, 2015).

Corroborando isso, Prata (1992, p. 168) afirma que a transição epidemiológica se caracteriza "[...] pela evolução progressiva de um perfil de alta mortalidade por doenças infecciosas para um outro 
onde predominam os óbitos por doenças cardiovasculares, neoplasias, causas externas e outras doenças consideradas crónico-degenerativas". Essa transição é caracterizada por complexas alterações nos padrões saúde-doença e em suas interações sob influência de fatores demográficos, econômicos e sociais, os quais seriam determinantes e consequentes. Assim, uma das características do processo de transição epidemiológica é o aumento da incidência de doenças crônicas não transmissíveis, as quais surgiram com maior impacto em países desenvolvidos, mas que, a partir da década de 1960, espalha-se rapidamente pelo Brasil (PEREIRA; ALVES-SOUZA; VALE, 2015).

Para Schramm et al. (2004, p. 898), "O conceito de transição epidemiológica tem merecido críticas pelo fato de a transformação dos padrões de saúde não obedecer aos mesmos parâmetros na sequência, intensidade e velocidade, em diferentes regiões". Lebrão (2007) pontua que a transição epidemiológica na América Latina conta com uma especificidade central se comparada aos países desenvolvidos. Segundo a autora, nos países latino-americanos, o aumento da expectativa de vida chegou antes da melhoria das condições econômicas e sociais dos países e das populações. Teríamos uma população mais pobre vivendo mais tempo (em decorrência da revolução da medicina em meados do século $X X$ ), diferentemente dos países desenvolvidos, onde houve, em um primeiro momento, a melhoria econômica e social das nações e, consequentemente, das pessoas, para depois se alcançar uma maior expectativa de vida. Assim, compreender esse processo seria fundamental para o entendimento da dinâmica atual da relação saúde-doença na região.

Neste viés, Schramm et al. (2004), apoiados na ideia de Chaimowicz (1997), apresentam que as alterações nos padrões demográficos (transição demográfica) mantêm relação direta com transição epidemiológica, pois a diminuição dos óbitos na população mais jovem, geralmente ligados às doenças infecciosas, proporcionou que essa parcela da população passasse a conviver com as doenças crônico-degenerativas. Ao passo que cresce o número de idosos, seria mais frequente a ocorrência de doenças não transmissíveis.

Sobre a transição demográfica, Lebrão (2007) apresenta que a transição epidemiológica e a transição demográfica não são a mesma coisa, porém mantêm íntima relação ao passo que se relacionam e se modificam. Assim, a alteração nos padrões de morbi/mortalidade impacta no aumento ou não da expectativa de vida, demograficamente, da mesma forma que as variações demográficas (aumento da expectativa de vida ou menor fecundidade) atuam na alteração dos padrões de morbi/mortalidade.

No contexto brasileiro, Prata (1992) pontua que a transição epidemiológica no perfil de mortalidade, das doenças infecciosas para as cardiovasculares, neoplasias e causas externas, ocorre com certa persistência, embora não se apresentem como um padrão geral, havendo incidência e prevalência diferentes em cada região do país. Da mesma forma, pontua que a incidência e prevalência das doenças infecciosas encontram-se nas regiões de infraestrutura mais precária, entre a população mais pobre e onde as desigualdades sociais são mais relevantes.

Nesse viés, tem-se que o declínio do impacto das doenças infecciosas, em todos os níveis, deve-se, sobretudo, à melhora na cobertura do saneamento básico, à melhoria nas condições de habitação, à introdução da vacinação e ao avanço dos fármacos como os antibióticos (WALDMAN; SATO, 2016).

A relação percebida entre a questão sanitária e o perfil de mortalidade advém do próprio processo histórico de organização do Brasil, no qual a evolução nos indicadores sanitários encontra grande barreira nas próprias políticas de planejamento e de saúde, tanto nacional, quanto localmente.

Pereira, Alves-Souza e Vale (2015) pontuam que a transição também deve ser analisada sob o prisma da organização social e da atenção voltada às ações de prevenção em saúde, pois o desenvolvimento econômico e social colabora com a transição ao passo que possibilita o desenvolvimento individual e coletivo, de ações sanitárias, saneamento, da mesma forma em que a atenção preventiva tende a estar cada vez mais voltada às doenças crônicas e à população de faixa etária mais avançada. No entanto, Lebrão (2007), ao se referir ao envelhecimento da população brasileira, salienta que devemos mudar o paradigma de que pessoas idosas devem ser vistas como sinal de problema de saúde. Pelo contrário, devem-se buscar meios para que as mesmas possam ter maior qualidade de vida, citando como exemplo de quebra desse paradigma o termo "envelhecimento ativo" criado pela Organização Mundial da Saúde (OMS).

Contudo, no contexto brasileiro, a transição aqui identificada possui uma especificidade: como a reemergência de doenças infecciosas e parasitárias (se não com alto índice de mortalidade, com alto índice de morbidade - processo de afastamento do bem-estar fisiológico ou psicológico, DOl:http://dx.doi.org/10.14393/Hygeia153447153 Hygeia 15 (34): $130-143$, Dez./2019 página 133 
dimensionado objetivamente ou relatado de forma subjetiva), bem como o aumento da incidência das causas externas e de violência social de todas as formas não aferem um padrão de transição epidemiológico homogêneo, a distribuição dos riscos e agravos é diferente a depender da região do país (PEREIRA; ALVES-SOUZA; VALE, 2015).

Schramm et al. (2004) afirma que, no Brasil, a transição epidemiológica não ocorre de acordo com o modelo experimentado pela maior parte dos países industrializados, diferindo até mesmo dos vizinhos da América Latina, como Cuba, Chile e Costa Rica.

Dessa forma, tem-se destacado que a transição epidemiológica se relaciona com as ações e alterações nos padrões de vida, mudanças demográficas e sanitárias, em âmbitos local, nacional e global. Logo, como os agentes que contribuem com alteração no perfil de mortalidade não se apresentam de maneira homogênea em todas as escalas, a própria transição pode ocorrer de maneira desigual em uma mesma região ou país.

Um parâmetro sobre o contexto das doenças infecciosas, no cenário nacional, pode ser observado em Waldman e Sato (2016). Os autores apresentam que as doenças infecciosas com forte tendência de declínio são as endemias rurais (malária, esquistossomose mansônica e doença de Chagas), as doenças diarreicas e as doenças imunopreveníveis (como sarampo, rubéola, poliomielite e varíola). Da mesma forma, apresentam o grupo de doenças infecciosas com tendência de declínio moderado: tuberculose e hanseníase. Consequentemente, o grupo de doenças reemergentes: tracoma, leishmaniose visceral, cólera, febre amarela e dengue; e de doenças infecciosas emergentes: Aids, Chikungunya e Zika.

Sendo assim, esses aspectos apresentados apontam para a importância de um olhar heterogêneo na questão epidemiológica brasileira, pois o padrão de doenças jamais pode ser considerado estabelecido por completo, da mesma forma que as doenças controladas ou erradicadas não podem deixar de ser objeto de vigilância permanente.

No Brasil, as diferentes regiões do território podem apresentar diferentes situações frente à transição epidemiológica, havendo casos de transição já concluída e outros ainda em curso (PRATA, 1992).

Nesse viés:

Dessa forma, apresenta-se uma complexa diversificação na situação epidemiológica devido à distribuição irregular dos riscos e agravos em cada população, onde diferentes regiões do país apresentam taxas, coeficientes e situações desiguais, ou até mesmo em microrregiões do mesmo estado, da mesma cidade, demonstrando assim, diferenças importantes nos diversos grupos populacionais em relação às condições de vida e trabalho no país (PEREIRA; ALVES-SOUZA; VALE, 2015, p. 102).

Face ao exposto, observam-se no contexto do estado de Mato Grosso do Sul, a partir do recorte temporal definido (2007 a 2017), dois grupos de doenças (CID-10: algumas doenças infecciosas; e parasitárias e do grupo de neoplasias) que poderão dar um parâmetro do perfil da transição epidemiológica do estado sul-mato-grossense, quantificando o declínio ou aumento dos óbitos em cada grupo.

\section{A CLASSIFICAÇÃO CID-10 QUANTO AO GRUPO DE ALGUMAS DOENÇAS INFECTO PARASITÁRIAS E DO GRUPO DAS NEOPLASIAS}

O CID-10 é uma classificação internacional, a qual padroniza e cataloga as doenças e demais problemas de saúde, tendo como referência a nomenclatura internacional de doenças estabelecida pela OMS (Organização Mundial de Saúde). Possibilita que programas e sistemas possam referenciar as classificações de forma padronizada, possibilitando que, em todo o território nacional, sejam padronizadas a morbidade e a mortalidade hospitalar e ambulatorial (DATASUS, 2018).

O capítulo I do CID-10 é composto por aproximadamente duzentas doenças, divididas em vinte e um subconjuntos. Dessa forma, por uma questão de demasiada quantidade de doenças, apresentamos, no Quadro 1 a seguir, os subconjuntos da CID-10 A00-B99 (Algumas doenças infecciosas e parasitárias). 
Quadro 1 - Classificação do capítulo I CID-10, (A00-B99) algumas doenças infecciosas e parasitárias, subconjuntos.

\begin{tabular}{|l|l|l|}
\hline $\begin{array}{l}\text { A00-A09 Doenças infecciosas } \\
\text { intestinais }\end{array}$ & A15-A19 Tuberculose & $\begin{array}{l}\text { A20-A28 Algumas doenças } \\
\text { bacterianas zoonóticas }\end{array}$ \\
\hline $\begin{array}{l}\text { A30-A49 Outras doenças } \\
\text { bacterianas }\end{array}$ & $\begin{array}{l}\text { A50-A64 Infecções de transmissão } \\
\text { predominantemente sexual }\end{array}$ & $\begin{array}{l}\text { A65-A69 Outras doenças por } \\
\text { espiroquetas }\end{array}$ \\
\hline $\begin{array}{l}\text { A70-A74 Outras doenças } \\
\text { causadas por clamídias }\end{array}$ & A75-A79 Rickettsioses & $\begin{array}{l}\text { A80-A89 Infecções virais do } \\
\text { sistema nervoso central }\end{array}$ \\
\hline $\begin{array}{l}\text { A90-A99 Febres por arbovírus e } \\
\text { febres hemorrágicas virais }\end{array}$ & $\begin{array}{l}\text { B00-B09 Infecções virais } \\
\text { caracterizadas por lesões de pele e } \\
\text { mucos }\end{array}$ & B15-B19 Hepatite viral \\
\hline $\begin{array}{l}\text { B20-B24 Doenças pelo vírus da } \\
\text { imunodeficiência humana [HIV] }\end{array}$ & B25-B34-Outras doenças por vírus & B35-B49 Micoses \\
\hline $\begin{array}{l}\text { B50-B64 Doenças devidas a } \\
\text { protozoários }\end{array}$ & B65-B83 Helmintíases & $\begin{array}{l}\text { B85-B89 Pediculose, acaríase e } \\
\text { outras infestações }\end{array}$ \\
\hline $\begin{array}{l}\text { B90-B94 Sequelas de doenças } \\
\text { infecciosas e parasitárias }\end{array}$ & $\begin{array}{l}\text { B95-B97 Agentes de infecções } \\
\text { bacterianas, virais e outros agentes }\end{array}$ & B99 Outras doenças infecciosas \\
\hline
\end{tabular}

Fonte: Brasil (2018).

Da mesma forma, o capítulo II da classificação destina-se ao CID-10 C00-D48 Neoplasias (tumores), o qual tem em torno de cento e quarenta doenças divididas em dezoito subconjuntos, que podem ser visualizados no Quadro 2.

Quadro 2 - Classificação do capítulo II CID-10, (C00-D48) Neoplasias (tumores), subconjuntos.

\begin{tabular}{|l|l|l|}
\hline $\begin{array}{l}\text { C00-C14 Neoplasias [tumores] } \\
\text { malignas(os) do lábio, cavidade } \\
\text { oral e faringe }\end{array}$ & $\begin{array}{l}\text { C15-C26 Neoplasias [tumores] } \\
\text { malignas(os) dos órgãos digestivos }\end{array}$ & $\begin{array}{l}\text { C30-C39 Neoplasias [tumores] } \\
\text { malignas(os) do aparelho } \\
\text { respiratório e dos órgãos } \\
\text { intratorácicos }\end{array}$ \\
\hline $\begin{array}{l}\text { C40-C41 Neoplasias [tumores] } \\
\text { malignas(os) dos ossos e das } \\
\text { cartilagens }\end{array}$ & $\begin{array}{l}\text { C43-C44 Melanoma e outras(os) } \\
\text { neoplasias [tumores] malignas(os) } \\
\text { da pele }\end{array}$ & $\begin{array}{l}\text { C45-C49 Neoplasias [tumores] } \\
\text { malignas(os) do tecido mesotelial e } \\
\text { tecidos moles }\end{array}$ \\
\hline $\begin{array}{l}\text { C50 Neoplasias [tumores] } \\
\text { malignas(os) da mama }\end{array}$ & $\begin{array}{l}\text { C51-C58 Neoplasias [tumores] } \\
\text { malignas(os) dos órgãos genitais } \\
\text { femininos }\end{array}$ & $\begin{array}{l}\text { C60-C63 Neoplasias [tumores] } \\
\text { malignas(os) dos órgãos genitais } \\
\text { masculinos }\end{array}$ \\
\hline $\begin{array}{l}\text { C64-C68 Neoplasias [tumores] } \\
\text { malignas (os) do trato urinário }\end{array}$ & $\begin{array}{l}\text { C69-C72 Neoplasias [tumores] } \\
\text { malignas(os) dos olhos, do } \\
\text { encéfalo e de outras partes do } \\
\text { sistema nervoso central }\end{array}$ & $\begin{array}{l}\text { C73-C75 Neoplasias [tumores] } \\
\text { malignas(os) da tireóide e de } \\
\text { outras glândulas endócrinas }\end{array}$ \\
\hline $\begin{array}{l}\text { C76-C80 Neoplasias [tumores] } \\
\text { malignas(os) de localizações mal } \\
\text { definidas, secundárias e de } \\
\text { localizações não especificadas }\end{array}$ & $\begin{array}{l}\text { C81-C96Neoplasias [tumores] } \\
\text { malignas(os) do tecido linfático, } \\
\text { hematopoético e tecidos correlatos }\end{array}$ & $\begin{array}{l}\text { C97 Neoplasias [tumores] } \\
\text { malignas(os) de localizações } \\
\text { múltiplas }\end{array}$ \\
\hline $\begin{array}{l}\text { D00-D09 Neoplasias [tumores] in } \\
\text { situ }\end{array}$ & $\begin{array}{l}\text { D10-D36 Neoplasias [tumores] } \\
\text { benignas(os) }\end{array}$ & $\begin{array}{l}\text { D37-D48 Neoplasias [tumores] de } \\
\text { comportamento incerto ou } \\
\text { desconhecido }\end{array}$ \\
\hline
\end{tabular}

Fonte: Brasil (2018).

Logo, esses dois importantes grupos abarcam as doenças que foram consideradas nesta análise, não de forma individualizada, mas de todo o grupo disposto em cada capítulo. 


\section{CONTEXTUALIZAÇÃO GEOGRÁFICA DO ESTADO DE MATO GROSSO DO SUL}

Mato Grosso do Sul está localizado na região Centro-Oeste do Brasil. O estado sul-mato-grossense é o $6^{\circ}$ do país em área territorial, possuindo $357.145,531 \mathrm{~km}^{2}$ (ano de 2016). A população contabilizada no último censo (2010) foi de 2.449.024 pessoas, a projeção para o ano de 2017 foi de 2.713 .147 habitantes. Quanto à densidade demográfica e à população residente por situação domiciliar (urbana/rural), também contabilizada no último censo (2010), foi de $6,86 \mathrm{hab} / \mathrm{km}^{2} ; 2.097 .238 \mathrm{e}$ 351.786, respectivamente (IBGE, 2018). A Figura 1 mostra a localização do estado de Mato Grosso do Sul.

As condições climáticas de Mato Grosso do Sul em grande parte se assemelham às da região Centro-Oeste do Brasil. Segundo o Macrozoneamento desse estado, ocorrem dois tipos de clima: o tropical úmido com estação chuvosa no verão e seca no inverno; e o mesotérmico úmido sem estiagem, em que a temperatura do mês mais quente é superior a $22^{\circ} \mathrm{C}$, apresentando no mês mais seco uma precipitação superior a $30 \mathrm{~mm}$ de chuva (MATO GROSSO DO SUL, 1989).

Figura 1- Divisão político-administrativa do estado de Mato Grosso do Sul e sua localização no território brasileiro.

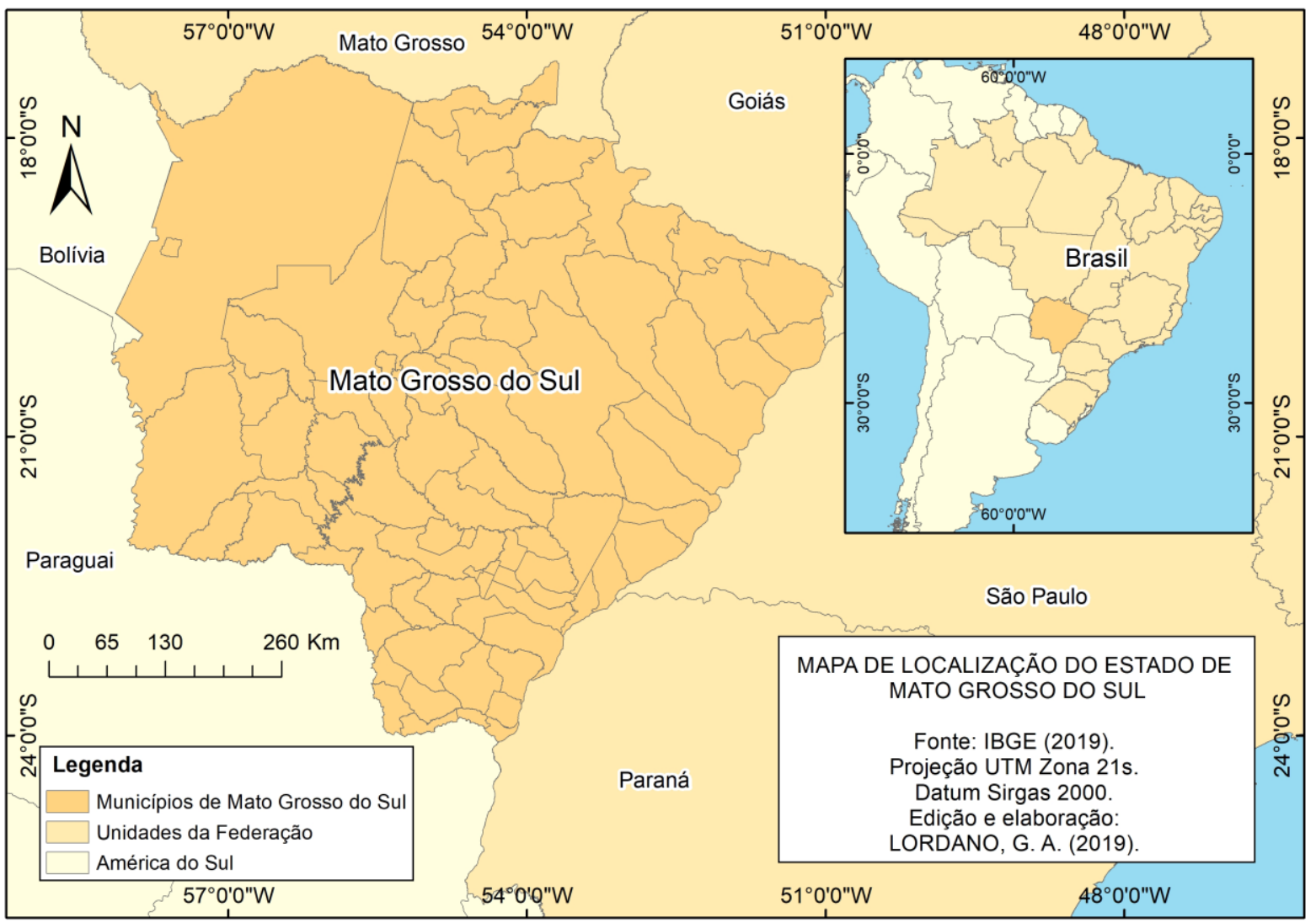

Fonte: IBGE (2019). Elaboração: LORDANO, G. A. (2019).

Mostra-se oportuno, no contexto desta pesquisa, um assunto que não nos deixa calar: o constante crescimento do uso de agrotóxicos em Mato Grosso do Sul. Um trabalho realizado pelo Ministério da Saúde intitulado "Diretrizes Nacionais para a Vigilância em Saúde de Populações Expostas a Agrotóxicos" chama a atenção para o consumo de agrotóxicos e o seu então aumento no Brasil, além das consequências que o mesmo pode causar aos seres humanos e ao meio ambiente (BRASIL, 2017). 
Em estudo realizado por Bombardi (2017), a autora, utilizando-se de dados do Sindicato Nacional da Indústria de Produtos para a Defesa Vegetal (SINDIVEG), esclarece que as culturas de soja, milho e cana, no ano de 2015 , foram responsáveis por $72 \%$ de todo o agrotóxico comercializado.

Salienta-se que o Brasil é um grande consumidor de agrotóxicos e, para referenciarmos nossa fala, Brasil $(2017$, p. 7) destaca que "Em 2008, o Brasil ocupou a primeira posição no ranking mundial de países consumidores de agrotóxicos [...]".

Bombardi (2017), utilizando-se de dados do Instituto Brasileiro do Meio Ambiente e dos Recursos Naturais Renováveis (IBAMA) para a busca de dados referentes ao uso de agrotóxicos no Brasil, mostra que em 2000 o total de agrotóxicos usados era cerca de 170.000 toneladas. Já no ano de 2014 o consumo foi de 500.000 toneladas.

Retratando-se nosso objeto de estudo, o estado de Mato Grosso do Sul, podemos verificar que o mesmo também tem contribuído na participação do uso de agrotóxicos, pois vem se destacando no cenário do agronegócio.

Para compreendermos como se deu o crescimento desse cenário no estado, cabe aqui tecer algumas considerações: após ser desmembrado de Mato Grosso (uno), Mato Grosso do Sul tinha uma característica econômica que se pautava no setor primário, sendo que a pecuária ocupava o grande destaque. Porém, mesmo assim, não possuía uma alta produção, pois os criadores afirmavam que, com a pouca valorização do gado, não era possível realizar investimentos. Sendo assim, o governo preocupou-se em criar programas de financiamento a fim de melhorar a situação produtiva sul-matogrossense (EMBRATER, 1977).

Entre os programas criados cabe destacar "o Programa de Desenvolvimento dos Cerrados (POLOCENTRO), que foi instituído pelo Decreto 75.320, de 29.01.75, com o objetivo de incorporar ao processo produtivo da agropecuária extensa área de cerrados do Brasil Central" (EMBRATER, 1977, p. 63). Sendo assim, realizou-se a supressão da vegetação e, por conseguinte, a introdução de culturas com a utilização dos mais diversos tipos de agrotóxicos.

Silva (2010), retratando a área dos cerrados, salienta que foi a partir de 1970 que começou o seu processo de transformação, mostrando ainda que:

O processo de interiorização e o avanço sob a região do setor agropecuário e, posteriormente, do sulcroalcooleiro, transforma nas décadas subsequentes todo o território e, por consequência, o Cerrado passa dar lugar a atividades econômicas intensivas cujas bases estão na exportação de commodities (SILVA, 2010, p. 45).

Além dessas alterações reportadas por Silva (2010) a respeito do cerrado, é também perceptível o avanço da agricultura da soja no Pantanal em Mato Grosso do Sul, podendo acarretar sérios problemas a esse rico bioma.

Atualmente, é recorrente vermos em noticiários as mais diversas consequências que os agrotóxicos têm causado tanto aos seres humanos como ao próprio meio em que vivemos. Como exemplo, trazemos aqui os primeiros cinco municípios de Mato Grosso do Sul que se destacaram nos índices de intoxicação por uso de agrotóxicos (1: 100.000 habitantes), como mostra a Figura 2.

Esses dados nos trazem uma ideia e a preocupação do intensivo uso de agrotóxicos nas lavouras, prejudicando a saúde da população, que irá necessitar de tratamentos médicos, e do próprio meio em si, que também pode sofrer sérias consequências, acarretando os mais diversos recursos. Segundo Bombardi (2017) o uso de agrotóxicos é utilizado não só nas plantações. Como exemplo disso, a autora cita o Malationa, um inseticida utilizado para combater alguns vetores, como dengue e Zika. Segundo Bombardi (2017, p. 34), [...] "para o período de 2012 a 2014 a média de agrotóxicos no Brasil foi de $8,33 \mathrm{~kg}$ por hectare. Nos estados de Mato Grosso, Mato Grosso do Sul, Goiás e São Paulo, este número figura entre 12 e $16 \mathrm{~kg}$ por hectare".

Outro ponto que se apresenta como fundamental nessa contextualização, face à pressão que exerce na alteração do perfil da mortalidade em todas as escalas, é o atendimento da população com serviços de saneamento. Logo, mostram-se breves considerações sobre o atendimento à população quanto ao abastecimento de água e rede de esgoto. 
Figura 2- Mapa do estado de Mato Grosso do Sul, demonstrando dados sobre os índices de intoxicação pelo uso de agrotóxicos, no período de 2007-2014.

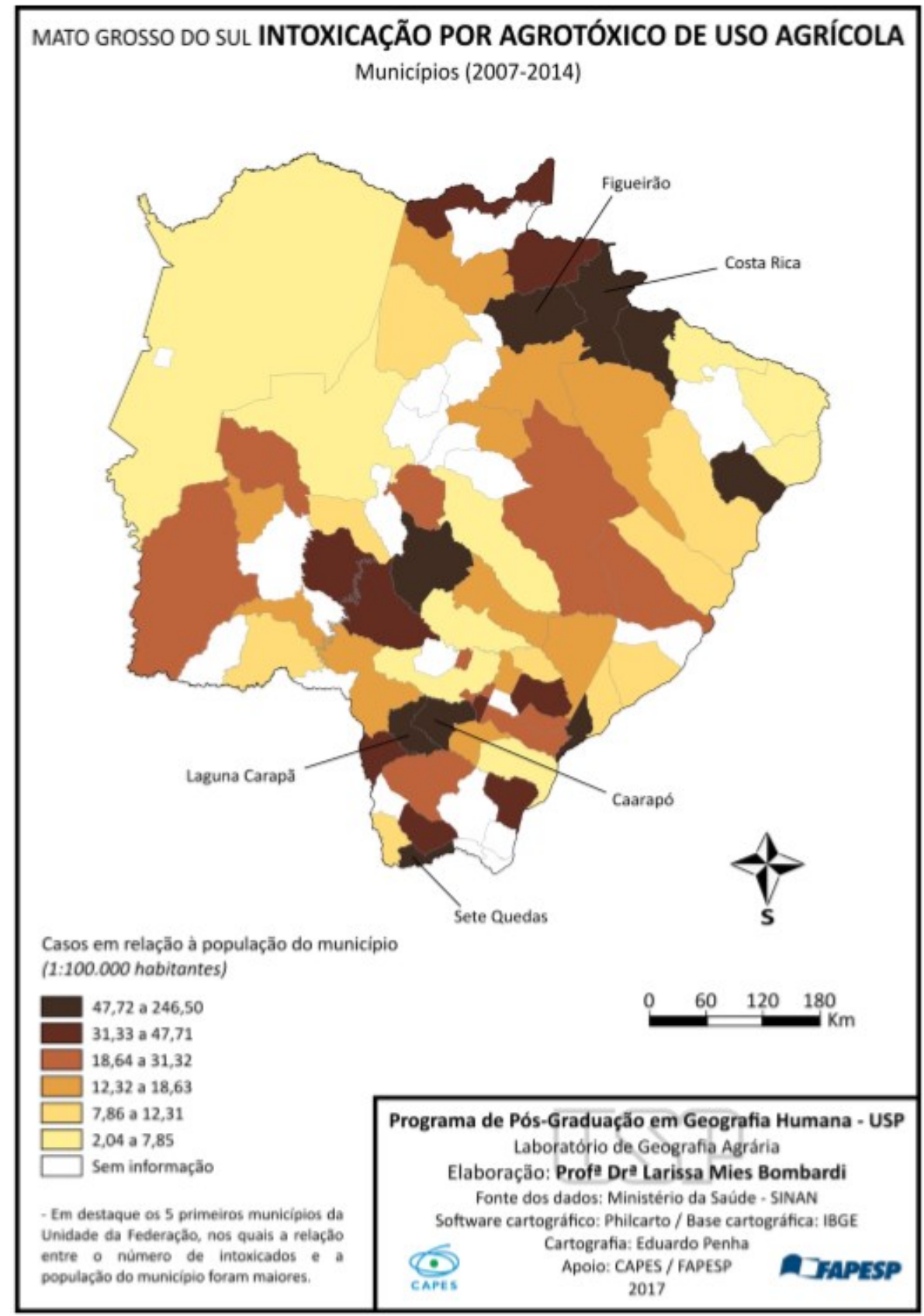

Fonte: Bombardi (2017, p. 133).

Segundo o Diagnóstico dos Serviços de Água e Esgotos, divulgado em 2016 pela Secretaria Nacional de Saneamento Ambiental (SNSA) do Ministério das Cidades (MCidades), que utiliza como fonte de dados o Sistema Nacional de Informações sobre Saneamento (SNIS), no que diz respeito ao atendimento da população urbana por rede de água, Mato Grosso do Sul possui um percentual de mais de $90 \%$ e, para atendimento da população urbana por rede de coletas de esgoto, o estado sulmato-grossense apresenta percentuais que variam entre $40 \%$ a $70 \%$. Tal estudo mostra que esses dados são relativos aos prestadores de serviços que participaram do Sistema Nacional de Informações sobre Saneamento (SNIS), em 2016, e não esclarece a respeito do total de esgoto coletado, a porcentagem de tratamento do mesmo. 
Salienta-se a importância de estudos relativos ao saneamento básico voltado à população das zonas rurais que, na maioria das vezes, realiza por conta própria o seu "saneamento", por meio da construção de fossas sépticas e da perfuração de poços. Esses feitos, dependendo da maneira como são utilizados, podem acarretar problemas de saúde a essa população.

\section{RESULTADOS E DISCUSSÃO}

Considerando a metodologia adotada, seguem os dados e suas respectivas análises.

Os dados coletados sobre a população do estado revelam que a estimativa populacional para o Mato Grosso do Sul era de 2.265.274 habitantes, em 2007, e de 2.713.147, em 2017, um aumento de $19,77 \%$ da população total no período (IBGE, 2007, 2018). Quanto ao número total de óbitos, por qualquer causa, no mesmo período, houve um aumento de $20,29 \%$ nos números absolutos, passando de 13.645 óbitos para 16.413 óbitos, em 2017. Quando considerado o coeficiente por 100 $\mathrm{mil} / \mathrm{hab}$., o aumento foi de $0,43 \%$ no mesmo período, passando de 602,35 óbitos para 604,94 óbitos por grupo de $100 \mathrm{mil} / \mathrm{hab}$.

Quanto aos óbitos causados por doenças dos dois grupos do escopo desta pesquisa, os dados da série histórica apresentam que, entre 2007 e 2017, os óbitos no estado de Mato Grosso do Sul causados por doenças do grupo CID-10, A00-B99 (Algumas doenças infecciosas e parasitárias), passaram de 579 óbitos para 627 óbitos em 2017, mostrando uma variação positiva de $8,30 \%$. Contudo, quando considerado o coeficiente de óbitos por 100mil/hab. para o período, percebe-se uma redução de $-9,59 \%$, indo de 25,55 para 23,10 óbitos a cada grupo de $100 \mathrm{mil} / \mathrm{hab}$. Quanto às mortes decorrentes das doenças pertencentes ao grupo CID-10, A00-B99 (Algumas doenças infecciosas e parasitárias), a variação positiva nos números absolutos de óbitos é da ordem de 48,12\%, variando de 1912 casos em 2007 para 2832 casos em 2017, e de 23,67\%, quando considerado o coeficiente de óbitos por 100mil/hab., de 84,40 em 2007 para 104,38 óbitos a cada grupo de 100mil/hab. em 2017, como pode ser observado na Tabela 1.

Tabela 1 - Série histórica da mortalidade por doenças pertencentes ao grupo CID-10, A00-B99 (Algumas doenças infecciosas e parasitárias) e CID-10 C00-D48 (Neoplasias) no estado de Mato Grosso do Sul, 2007 a 2017.

\begin{tabular}{ccccc}
\hline & \multicolumn{2}{c}{$\begin{array}{c}\text { CID-10, A00-B99 } \\
\text { ANO }\end{array}$} & $\begin{array}{c}\text { CID-10 C00-D48 } \\
\text { (Neoplasias) }\end{array}$ \\
\cline { 2 - 5 } (Algumascitas infecciosas e & Coef. & n. & Coef. \\
\hline 2007 & n. & 25,55 & 1912 & 84,40 \\
2008 & 579 & 23,46 & 2158 & 92,75 \\
2009 & 546 & 22,86 & 2138 & 89,53 \\
2010 & 605 & 25,00 & 2244 & 91,62 \\
2011 & 521 & 20,95 & 2214 & 89,03 \\
2012 & 601 & 23,80 & 2374 & 94,03 \\
2013 & 586 & 22,87 & 2398 & 93,59 \\
2014 & 553 & 21,26 & 2537 & 97,57 \\
2015 & 602 & 22,82 & 2577 & 97,69 \\
2016 & 667 & 24,93 & 2704 & 101,06 \\
2017 & 627 & 23,10 & 2832 & 104,38 \\
\hline
\end{tabular}

${ }^{*}$ coeficientes por 100.000 hab.

Fonte: IBGE (2007, 2018); Mato Grosso do Sul (2018). 
Verificou-se, assim, que os óbitos relacionados às doenças pertencentes aos CID-10, A00-B99 (Algumas doenças infecciosas e parasitárias) possuem redução no coeficiente de mortalidade e, nos óbitos causados pelas doenças relacionadas às neoplasias, CID-10 C00-D48, um aumento no período.

Tabela 2 - Tendência da mortalidade por doenças pertencentes ao grupo CID-10, A00-B99 (Algumas doenças infecciosas e parasitárias) e CID-10 C00-D48 (Neoplasias) no estado de Mato Grosso do Sul, 2007 a 2017.

\begin{tabular}{lcccccc}
\hline $\begin{array}{l}\text { Grupo de Doenças conforme a } \\
\text { classificação CID-10 }\end{array}$ & Média & Mediana & $\begin{array}{c}\text { Desvio } \\
\text { Padrão }\end{array}$ & R2 & P & $\begin{array}{c}\text { CV } \\
(\mathrm{DP} / \bar{x} * 100) \%\end{array}$ \\
\hline $\begin{array}{l}\text { CID-10, A00-B99 (Algumas } \\
\text { doenças infecciosas e parasitárias) }\end{array}$ & 23,30 & 23,10 & 1,42 & 0,05 & 0,42 & 6,11 \\
CID-10 C00-D48 (Neoplasias) & 94,15 & 93,59 & 5,72 & 0,83 & 0,00 & 6,08 \\
\hline
\end{tabular}

Fonte: IBGE (2007, 2018); Mato Grosso do Sul (2018).

Na Tabela 2 e nas Figuras 3 e 4, pode ser visualizada a tendência dos coeficientes de mortalidade padronizados por meio do método de regressão linear simples. Quanto à mortalidade por doenças pertencentes ao grupo CID-10, A00-B99 (Algumas doenças infecciosas e parasitárias), o $\mathrm{R}^{2}$ apresentou fraco ajustamento de amostra $(0,05), p>0,05$, não significativo e coeficiente de variação (CV) com $6,11 \%$, indicando baixa dispersão dos dados. Já a mortalidade relacionada às doenças do grupo CID-10 C00-D48 (Neoplasias) apresenta significativa tendência de aumento. $O R^{2}$ indica o alto ajustamento da amostra $(0,83), p<0,05$, altamente significativo, e coeficiente de variação $(\mathrm{CV}) \mathrm{com}$ $6,08 \%$ indicando baixa dispersão dos dados.

Figura 3 - Tendência dos coeficientes da mortalidade por doenças dos grupos CID-10, A00-B99

(Algumas doenças infecciosas e parasitárias) no estado de Mato Grosso do Sul, 2007-2017.

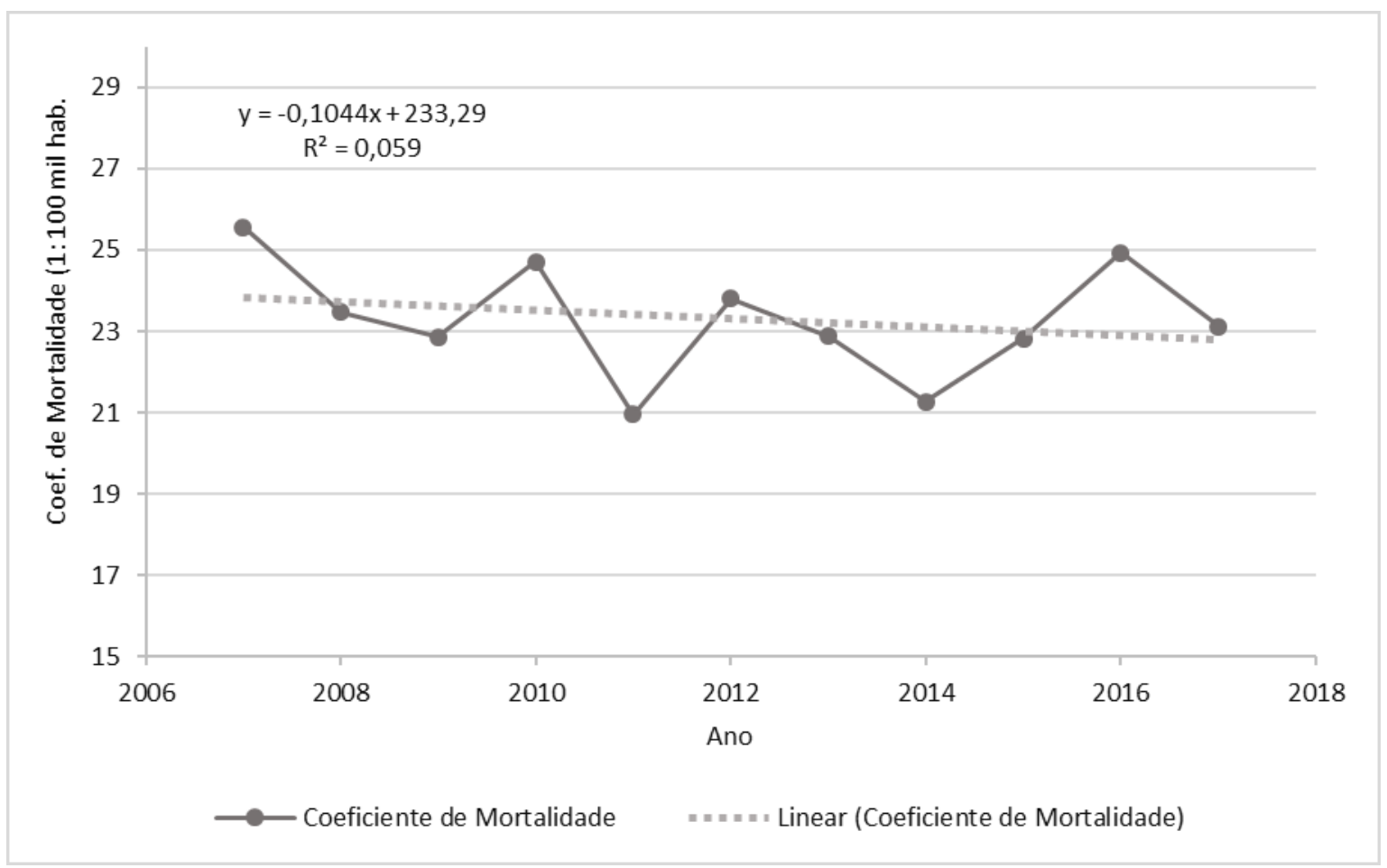

Fonte: IBGE (2007, 2018); Mato Grosso do Sul (2018). 
Figura 4 - Tendência dos coeficientes da mortalidade por doenças dos grupos CID-10 C00-D48 (Neoplasias) no estado de Mato Grosso do Sul, 2007-2017.

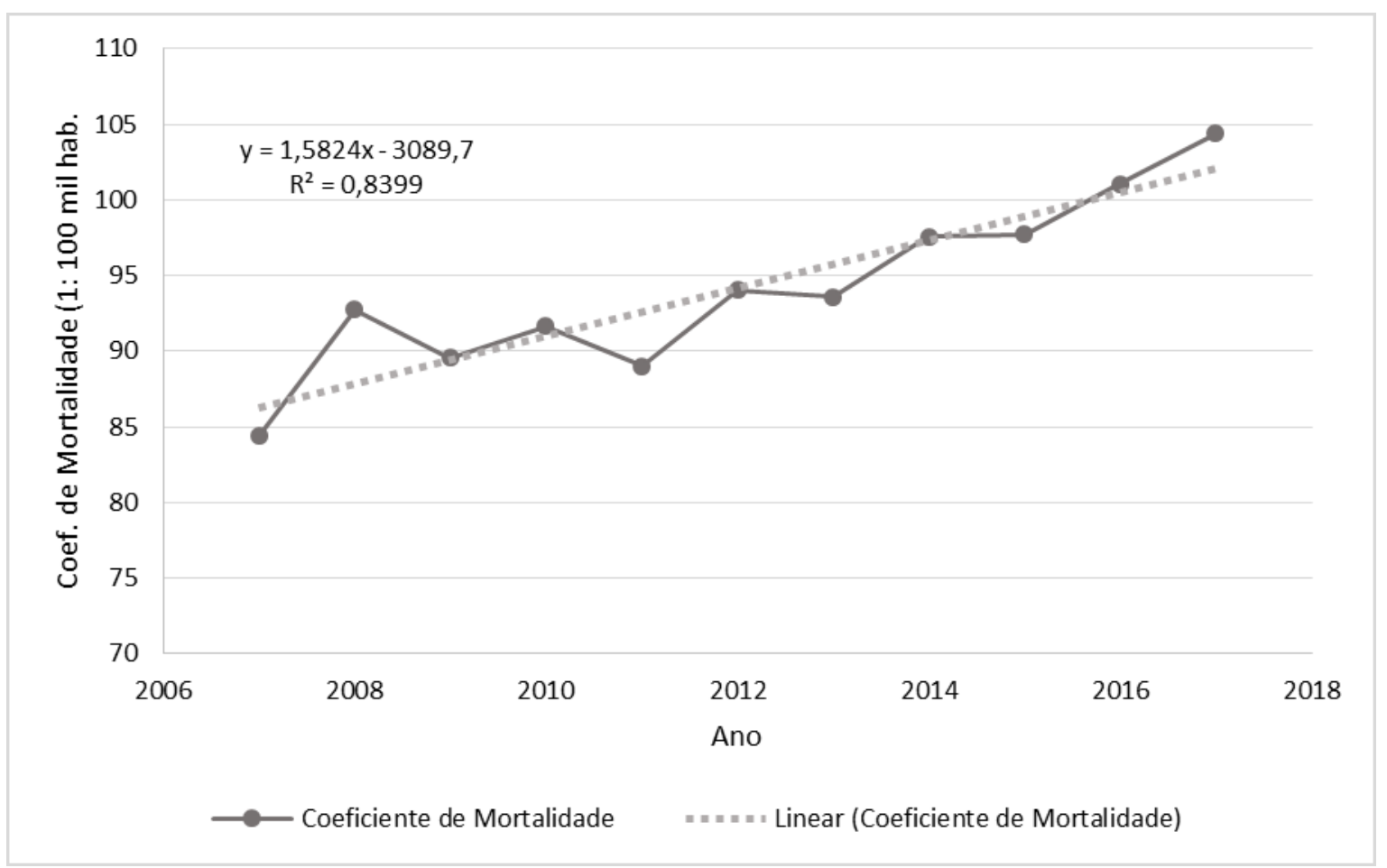

Fonte: IBGE (2007, 2018); Mato Grosso do Sul (2018).

Com base nos dados analisados, os óbitos no grupo das doenças do CID-10 A00-B99 (Algumas doenças infecciosas e parasitárias) não apresentam redução com significância estatística no período, apesar de chamar atenção a redução de $-9,59 \%$ do coeficiente de mortalidade, ou ainda, da porcentagem deste grupo dentre o total de óbitos no estado $(-9,90)$ considerado o período de 2007 a 2017.

O fato de a queda dos óbitos por doenças infecciosas e parasitárias não ser mais acentuada em Mato Grosso do Sul remete-nos à prevalência dessas doenças estarem relacionadas à infraestrutura precária e à falta de saneamento básico adequado (PRATA, 1992; WALDMAN; SATO, 2016). Esse fato leva à suposição de que talvez no Estado as políticas públicas voltadas a estas questões não tenham avançado suficientemente nos últimos anos ou, ainda, aliado a isso ou não, que deva ser considerado que a reemergência das doenças desse grupo seja uma realidade no cenário nacional (PEREIRA; ALVES-SOUZA; VALE, 2015).

Nos casos que se referem às neoplasias, CID-10 C00-D48, o aumento é evidente e, de certa forma, muito considerável. A variação é de $+23,67 \%$ no coeficiente de mortalidade e de $+23,13 \%$, quando considerado seu peso entre todas as causas de óbitos no estado. Assim, embasados em Schramm et al. (2004), essa alteração no perfil de saúde da população do estado fará com que ocorra um aumento na procura por serviços de saúde, havendo a necessidade de investimentos em aparatos tecnológicos que visem ao tratamento dos indivíduos, o que acarretará em gastos.

Nesse sentido, ressalta-se a importância de que as ações e políticas públicas estejam em consonância com as alterações ou estabilidade no perfil epidemiológico do estado, uma vez que elas devem ser pensadas, elaboradas e executadas visando não somente a oferecer o tratamento clínico em si, mas também sob a ótica da logística dos pacientes que serão atendidos, pois atendimentos mais complexos e especializados tendem a ser alocados em localidades de maior centralidade na rede urbana. 


\section{CONSIDERAÇÕES FINAIS}

A partir da análise, podem-se tecer algumas considerações, até certo ponto, importantes sobre o perfil da transição epidemiológica no estado de Mato Grosso do Sul.

A hipótese inicial foi confirmada. Percebe-se o movimento de diminuição coeficiente de mortalidade do grupo de algumas doenças infecciosas e parasitárias, comumente associadas a regiões menos desenvolvidas com condições sanitárias mais defasadas e condições sociais mais precárias, ainda que, no período, a variação não se prove estatisticamente significante. Por outro lado, tem-se o aumento significativo do coeficiente de mortalidade e dos casos totais por neoplasias, comumente associadas ao aumento da expectativa de vida e alterações de padrões de consumo, alimentares e de produção. Em Mato Grosso do Sul, a alteração do perfil epidemiológico no período, baseada nos dois grupos observados, é caracterizada, sobretudo, pelo aumento do coeficiente de mortalidade por neoplasias, com um nível de significância muito alto e uma redução bem menos significativa das doenças infecciosas e parasitárias.

O coeficiente total de óbitos (todas as causas) tem um padrão relativamente estável, com um pequeno aumento $(+0,43 \%)$ de 2007 para 2017, contrariando Prata (1992), para quem o declínio do coeficiente geral de mortalidade seria uma realidade.

Em uma hipótese final desta pesquisa, pode-se supor que o constante avanço nas campanhas de prevenção em saúde, como as de vacinação, e a melhoria dos aspectos sanitários atuaram na redução das mortes por doenças infecciosas e parasitárias. Da mesma forma que o aumento da expectativa de vida, os hábitos alimentares, o sedentarismo e o vertiginoso uso de agrotóxico na agricultura estadual tendem a contribuir com o forte aumento dos casos de neoplasias. Claro que, nesse contexto, deve ser considerada a possibilidade de que a melhoria no diagnóstico da doença tem favorecido que cada vez menos ocorra a omissão dos casos.

Portanto, frente à alteração no padrão de mortalidade no estado de Mato Grosso do Sul, torna-se evidente a necessidade de as ações de saúde regional e a alocação de recursos em saúde serem voltadas à crescente demanda de diagnóstico e tratamento dos casos de Neoplasias, além da indispensável atenção às ações preventivas. Sobre isso, Schramm et al. (2004, p. 907) afirmam que "O uso racional dos recursos disponíveis torna imprescindível em saúde pública a busca permanente de instrumentos que ajudem a tomada de decisões".

Portanto, entende-se que os objetivos deste trabalho foram alcançados e que eles fornecem importantes contribuições às reflexões aqui propostas, bem como a futuras pesquisas. Salienta-se que se fazem necessárias pesquisas futuras que busquem uma projeção de como os coeficientes desses dois grupos de doenças se comportarão no futuro e, sobretudo, considerando as variáveis que favoreçam a redução ou o aumento nos casos.

\section{REFERÊNCIAS}

BOMBARDI, Larissa Mies. Atlas. Geografia do Uso de Agrotóxicos no Brasil e Conexões com a União Europeia. São Paulo: FFLCH - USP, 2017. 296 p. ISBN:978-85-7506-310-1. Disponível em: $<$ https://drive.google.com/file/d/1ci7nzJPm_J6XYNkdv_rt-nbFmOETH80G/view>. Acesso em: 11 de jun.2018.

BRASIL. Ministério da Fazenda. Secretaria de Previdência. Página da web. 2018. Disponível em: <http://www.previdencia.gov.br/dados-abertos/estatsticas/tabelas-cid-10/>. Acesso em: 02 de jun. de 2018.

. Ministério da Saúde. Secretaria de Vigilância em Saúde. Departamento de Vigilância em Saúde Ambiental e Saúde do Trabalhador. Diretrizes nacionais para a vigilância em saúde de populações expostas a agrotóxicos. Brasília DF: Ministério da Saúde, 2017. Disponível em: <http://bvsms.saude.gov.br/bvs/publicacoes/diretrizes_vigilancia_populacoes_expostas_agrotoxicos.p df>. Acesso em: 12 de jun. 2018.

Ministério das Cidades. Secretaria Nacional de Saneamento Ambiental - SNSA. Sistema Nacional de Informações sobre Saneamento: Diagnóstico dos Serviços de Água e Esgotos - 2016. Brasília: SNSA/MCIDADES, 2018. 220 p. Disponível em: <http://www.snis.gov.br/diagnostico-agua-eesgotos/diagnostico-ae-2016>. Acesso em: 10 jun. 2018. 
DATASUS. Departamento de Informática do SUS. Página da web. 2018. Disponível em: $<$ http://datasus.saude.gov.br/sistemas-e-aplicativos/cadastros-nacionais/cid-10>. Acesso em: $01 \mathrm{de}$ jul. 2018.

EMBRATER. Empresa Brasileira de Assistência Técnica e Extensão Rural. Coordenadoria Regional II. Mato Grosso do Sul: subsídios para o diagnóstico do estado. Campo Grande, dezembro de 1977.

IBGE. Instituto Brasileiro de Geografia e Estatística. Contagem da População 2007. Rio de Janeiro. 2007. ISBN 978-85-240-3995. Disponível em:

<https://ww2.ibge.gov.br/home/estatistica/populacao/contagem2007/contagem.pdf >. Acesso em: 01 de mai. 2018.

Mato Grosso do Sul. 2018. Disponível em:

$\overline{<h t t p s: / / c i d a d e s . i b g e . g o v . b r / b r a s i l / m s / p a n o r a m a>. ~ A c e s s o ~ e m: ~} 02$ mai. 2018.

. Portal de mapas. Organização do território. Página web. 2019. Disponível em: https://tinyurl.com/yxbb3fop

LEBRÃO, M. L. O envelhecimento no Brasil: aspectos da transição demográfica e epidemiológica. São Paulo. Saúde Coletiva. Editora Bolina. v. 4, n. 17, p. 135-140. 2007. ISSN: 1806-3365. Disponível em: <https://www.nescon.medicina.ufmg.br/biblioteca/imagem/2559.pdf>. Acesso em: 21 de jul. 2018.

LIMA, S. do C.; GUIMARÃES, R. B. Determinação social no complexo tecno-patogênico informacional da malária. Revista Brasileira de Geografia Médica e da Saúde (HIGEYA). Uberlândia. v. 3, n. 5, Dez/2007. p. 58 - 77. ISSN: 1980-1726. Disponível em:

<http://www.seer.ufu.br/index.php/hygeia/article/view/16884/9301>. Acesso em: 26 de mar. 2018.

MATO GROSSO DO SUL. Macrozoneamento Geoambiental do Estado de Mato Grosso do Sul. Campo Grande, 1989. Disponível em: <https://biblioteca.ibge.gov.br/visualizacao/livros/liv84710.pdf>. Acesso em: 18 jun. 2018.

Secretaria de Estado de Saúde. Plataforma TabNet. Página Web. Disponível em:

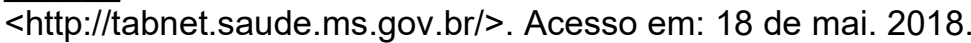

MICROSOFT OFFICE EXCEL. Software de edição de planilhas. Versão 2013. Microsoft Corporation.

OMRAM, A. R. The epidemiologic transition: a theory of the epidemiology of population change.

Bulletin of the World Health Organization. n. 79, v. 2 49, p. 161-170. 2001. Disponível em: <https://www.scielosp.org/article/ssm/content/raw/?resource_ssm_path=/media/assets/bwho/v79n2/v7 9n2a11.pdf>. Acesso em: 18 de jun. 2018.

PEREIRA, R. A; ALVES-SOUZA, R. A; VALE, J. de. S. O Processo de Transição Epidemiológica no Brasil: uma Revisão de Literatura. Revista Científica da Faculdade de Educação e Meio Ambiente (FAEMA). v. 6 n. 1. p. 99-108, jan/jun, 2015. ISSN 2179-4200. Disponível em:

<http://www.faema.edu.br/revistas/index.php/Revista-FAEMA/article/view/322>. Acesso em: 15 de mai. 2018.

PRATA, P. R. A Transição Epidemiológica no Brasil. Caderno de Saúde Pública. Rio de Janeiro, vol. 8 n. 2, p. 168-175, abr/jun, 1992. ISSN 1678-4464. Disponível em:

<http://www.scielo.br/pdf/csp/v8n2/v8n2a08.pdf >. Acesso em: 20 de mai. 2018. https://doi.org/10.1590/S0102-311X1992000200008

SCHRAMM, J. M. de A. et.al. Transição epidemiológica e o estudo de carga de doença no Brasil.

Revista Ciência \& Saúde Coletiva, v. 9, n. 4, p.897-908. 2004. ISSN: 1678- 4561. Disponível em: < http://www.scielo.br/pdf/csc/v9n4/a11v9n4.pdf>. Acesso em: 27 de jul. 2018. https://doi.org/10.1590/S1413-81232004000400011

SILVA, C. A. da. Pensar, fazer ciência e desafios da pesquisa em climatologia geográfica no CentroOeste. Mercator, Fortaleza, v. 9 n.1, p.39-51, Número Especial - dez. 2010. ISSN 1984-2201. Disponível em: <http://www.mercator.ufc.br/mercator/article/view/532>. Acesso em: 18 de jun. 2018.

WALDMAN, E. A; SATO, A. P. S. Trajetória das doenças infecciosas no Brasil nos últimos 50 anos: um contínuo desafio. Revista de Saúde Pública. São Paulo. v. 50 n. 68. 2016. ISSN 0034-8910. Disponivel em: <http://www.scielo.br/scielo.php?pid=S0034$89102016000100137 \&$ script=sci_abstract\&tIng=pt>. Acesso em: 15 de mai. 2018. 